\title{
Colopexia em ovinos da raça Dorper com prolapso retal
}

\author{
Colopexy in Dorper lambs with rectal prolapse
}

\section{Graziela Kopinits de Oliveira I Cassandra Kopinits de Oliveira ${ }^{\text {II }}$ Alceu Gaspar Raiser III Sildivane Valcácia Silva ${ }^{\text {IV }}$ Fernando Mônacov $^{\mathrm{V}}$}

\section{RESUMO}

Prolapso de reto é afecção comum em ovinos de cauda curta. Neste trabalho relata-se a técnica de colopexia para redução de prolapso retal em trinta ovinos da raça Dorper, dos quais, três vieram a óbito no período pós-operatório e três tiveram que ser sacrificados, pois além de apresentarem recidiva, um deles era idoso, e os outros três por se encontrarem bastante debilitados. Aos 15 dias após a cirurgia, cinco animais apresentaram recidiva do prolapso, sendo a colopexia refeita em três deles tendo bom resultado em apenas um, e os outros dois foram sacrificados. Aos 30 dias de pós-operatório um animal apresentou prolapso retal, os outros dezenove $(63,3 \%)$ estavam em adequado estado físico. A realização de colopexia é uma alternativa para o tratamento de prolapso retal em ovelhas da raça Dorper, porém recidivas e complicações são comuns.

Palavras-chave: prolapso retal, ovinos, Dorper, colopexia.

\section{ABSTRACT}

Rectal prolapse is a common affection in lambs of short tail. This study aimed at reporting the colopexy to reduce the rectal prolapse in Dorper lambs. Thirty animals were submitted to surgery and three of them died in the postoperative period. Three animals had to be sacrificed, because they have shown prolapse recurrence (one of them was old, and the other three were in a strong debilitated state). At 15 days after the surgery, five animals showed prolapse recurrence and the colopexy was performed again in three having good results in only one, the other two were sacrificed. At the 30 postoperative days, an animal showed rectal prolapse signals, the other nineteen animals (63,3\%) were healthy. The colopexy use is an option to rectal prolapse treatment in Dorper lambs, although, recurrences and complications are expected.

Key words: rectal prolapse, lambs, Dorper, colopexy.

\section{INTRODUÇÃO}

O rebanho ovino do nordeste é representado por população de, aproximadamente 6,7 milhões de animais, correspondente a $48,1 \%$ do rebanho nacional (IBGE, 1996), composto, em sua vasta maioria, por animais deslanados e semilanados (GUIMARÃES FILHO et al., 2000). A potencialidade do mercado de carne ovina é uma realidade, verificando-se que a demanda supera a oferta atualmente disponível, sendo que, para suprir a procura interna, o Brasil tem recorrido a importações da Nova Zelândia e do Uruguai. Com a agroindústria instalada e as tecnologias disponíveis, a ovinocultura brasileira poderá se destacar como atividade de grande impacto econômico (COLODO et al., 2004).

A Dorper é uma raça de ovino de tamanho médio, importada da África do Sul, resultado do cruzamento entre Dorset Horn e Blackhead Persian

\footnotetext{
IPrograma de Pós-graduação em Medicina Veterinária, Universidade Federal de Santa Maria (UFSM), Santa Maria, RS, Brasil. Endereço para correspondência: Rua Albert Bruce Sabin, n.02, apto 05, Universitário, 55016-535, Caruaru, PE, Brasil. E-mail: grakopinits@yahoo.com.br.

IIAutônoma, Recife, PE, Brasil.

IIIDepartamento de Clínica de Pequenos Animais, Centro de Ciências Rurais (CCR), UFSM, Santa Maria, RS, Brasil.

IVPrograma de Pós-graduação em Medicina Veterinária, Universidade Federal de Pernambuco (UFPE), Recife, PE, Brasil.

${ }^{\text {v} C u r s o ~ d e ~ M e d i c i n a ~ V e t e r i n a ́ r i a, ~ U n i a ̃ o ~ M e t r o p o l i n a ~ d e ~ E d u c a c ̧ a ̃ o ~(U N I M E), ~ L a u r o ~ d e ~ F r e i t a s, ~ B A, ~ B r a s i l . ~}$
} 
(SOUZA \& LEITE, 2000; NOTTER et al., 2004; BARROS et al., 2005) e possui aparente superioridade em relação à conformação e musculatura, sobre outras ovelhas lanadas. O peso médio dos adultos dessa raça varia entre 52 e 74kg (NOTTER et al., 2004). O Dorper pode resistir à desidratação e compensa rapidamente as perdas de peso, quando a água fica disponível, permitindo a animais dessa raça uma adaptação a regiões secas onde há limitação de água (CLOETE et al., 2000).

O prolapso do reto é afecção conhecida há muito tempo, sendo descrito pela primeira vez nos Papiros Eberes, em 1500 a.C. (CORMAN, 1993) e consiste na protrusão da sua mucosa pelo ânus. Pode ser classificado como parcial, quando somente a mucosa estiver envolvida, ou completo, situação em que toda a circunferência do órgão e todas as suas camadas estão prolapsadas (FOSSUM, 2005).

A cauda curta resulta numa maior incidência de prolapso de reto em ovinos, contudo, muitos criadores de ovelhas e produtores de carneiros para exposição realizam a caudectomia completa para criar a ilusão de maior musculatura na região pélvica, não convencidos dos efeitos negativos dessa técnica (THOMAS et al., 2003).

Em relação à genética, BULGIN (2006) acredita que, se um cordeiro for predisposto geneticamente ao prolapso, os ligamentos e estruturas internas não são fortes o bastante para manterem o intestino na área pélvica. De acordo com esse autor, a ocorrência de prolapso em animais com cortes de cauda curta se deve ao fato do músculo esfíncter anal se inserir nas primeiras quatro vértebras coccígeas, e quando elas são removidas, os músculos não têm onde se ancorar.

THOMAS et al. (2003) compararam em experimento realizado em seis localidades nos Estados Unidos, a prevalência de prolapso de reto em ovinos com o tamanho do corte da cauda. Foram realizados cortes de três comprimentos diferentes (curto, médio e longo) e os principais resultados foram que ovinos do sexo feminino tiveram maior incidência de prolapso do que os machos, e que os animais com corte de cauda curto foram os que apresentaram maior incidência desta afecção. Em relação à alimentação, dentre as localidades, apenas em duas havia alimentação diferenciada, em uma delas, onde maior número de animais apresentou o prolapso de reto, os alimentados apenas com ração tiveram maior prevalência que os alimentados com pasto sem suplementação de grãos.

De acordo com BULGIN (2006), o sexo afeta a ocorrência de prolapso, porque fêmeas possuem mais gordura interna, particularmente na pelve, que não é um tecido forte e lacera facilmente. Quando a ovelha tosse ou bali, a gordura não é capaz de sustentar o tecido retal e o intestino se insinua pelo ânus.

Segundo SCHOENIAN (2006), a correção do prolapso retal pode ser custo-proibitivo em animais, em regime de engorda e a matança imediata é geralmente recomendada para cordeiros de mercado, no entanto, para animais mais valiosos, o tratamento é recomendável. Se o prolapso for moderado, deve-se lavar e lubrificar o tecido exposto, reduzi-lo, realizandose sutura em bolsa de tabaco para manter o ânus parcialmente fechado em uma fixação temporária. Em casos mais graves, um anel de prolapso ou um segmento curto de mangueira pode ser usado para reparar o prolapso. O anel é introduzido no reto e uma faixa elástica é colocada ao redor da área a ser amputada. Como adjuvante, será necessário que o animal receba óleo mineral perianal, ou outro laxante para manter as fezes macias.

Para pequenos animais que possuem tecido viável, a redução do prolapso torna-se difícil em casos de protrusão extensa, ou após duas ou três tentativas malsucedidas de tratamento com a sutura de retenção, deve-se realizar a colopexia abdominal (MATTHIESEN \& MARRETA, 1998), ou seja, a fixação cirúrgica do cólon à parede abdominal (FOSSUM, 2005). Alguns autores preferem suturas, com ou sem prévia escarificação da camada serosa do cólon e da parede abdominal, enquanto outros procuram promover incisões nesses tecidos, com posterior aplicação de sutura entre as bordas correspondentes (BRUN et al., 2004).

Em cirurgias convencionais, está comprovado que tanto os fios absorvíveis como os não absorvíveis são adequados para a fixação do cólon. (FOSSUM, 2005). BRUN et al. (2004) realizaram estudo comparando o fio polipropileno com a poliglactina 910 na colopexia de cães, e verificaram que ambos são efetivos e resultaram em tempos semelhantes para a confecção das suturas. Contudo, a sutura com poliglactina 910 foi realizada com maior facilidade, sendo tal fato atribuído à menor memória que esse fio apresenta em relação ao propileno. $\mathrm{Na}$ análise histológica, observou-se que a deposição de tecido conjuntivo foi semelhante entre os grupos, sendo que, em todos os casos, o colágeno apresentava-se maturo.

Em relação à anestesia, SCOTT \& GESSERT (1997) relataram que a injeção epidural caudal de xilazina $\left(0,07 \mathrm{mg} \mathrm{kg}^{-1}\right)$ e lidocaína $\left(0,5 \mathrm{mg} \mathrm{kg} \mathrm{kg}^{-1}\right)$ combinadas promove analgesia efetiva e segura, permitindo restituição e retenção de prolapso retal, uterino ou da cérvice, no pós-parto de ovelhas.

Neste trabalho, o objetivo foi avaliar-se a prática de colopexia como tratamento de prolapso retal em ovinos da raça Dorper. 


\section{MATERIAL E MÉTODO}

Foram utilizados trinta ovinos da raça Dorper, sendo quatro machos e vinte e seis fêmeas, de propriedades dos Estados de Pernambuco, Paraíba e Rio Grande do Norte. Todos os animais foram diagnosticados clinicamente com prolapso retal (Figura 1A) e a redução cirúrgica foi realizada nos respectivos estados de origem.

Para a cirurgia, os animais foram submetidos a jejum alimentar de 24h e hídrico de, aproximadamente, $6 \mathrm{~h}$, lavados e tricotomizados em toda a região abdominal. Como anestesia, foi utilizado cloridrato de xilazina $^{\mathrm{a}}$, na dose de $0,5 \mathrm{mg} \mathrm{kg}^{-1}$, intramuscular e lidocaína ${ }^{\mathrm{b}} 2 \%$, via epidural, na dose de $100 \mathrm{mg}$ por animal. A antibioticoprofilaxia foi feita com gentamicinac, na dose de $4 \mathrm{mg} \mathrm{kg}^{-1}$, por via intramuscular, uma hora antes da realização da cirurgia.

Os animais foram colocados em cama operatória para ovinos e os membros locomotores amarrados tanto nas cirurgias a campo $(n=15)$, como naquelas efetuadas em sala cirúrgica $(\mathrm{n}=15)$ (FIGURA 1B). Após ser anestesiado e contido, cada animal foi submetido à anti-sepsia da região abdominal, com álcool-iodo-álcool, bem como à colocação dos panos de campo esterilizados.
Após redução manual do prolapso, um tubo falcon esterilizado foi inserido através do ânus e reto para facilitar a sua identificação. Mediante laparotomia retro-umbilical, o cólon foi localizado e uma porção de $6 \mathrm{~cm}$ foi escarificada com o bisturi (Figura 1C), bem como da parede abdominal, para posterior fixação.

Realizou-se então uma primeira linha de sutura de arrimo com fio poliglactina $910, n^{\circ} 0$, sendo que, em cada linha de sutura, foram aplicados, aproximadamente, cinco pontos. Após, o cólon foi deslizado em direção à linha média, para que a segunda linha de sutura fosse realizada, com o mesmo padrão de sutura, fio e quantidade de pontos (Figura 1D).

A cavidade abdominal foi lavada com $500 \mathrm{~mL}$ de solução de ringer com lactato sódico com objetivo de remover coágulos e possíveis contaminantes, sendo que em treze ovinos, essa solução não foi aspirada antes do fechamento da cavidade. A sutura da parede abdominal foi feita por pontos isolados simples, com fio poliglactina 910 , no 0 e o tecido subcutâneo com categute cromado $n^{-0} 0$, com padrão ziguezague. $\mathrm{Na}$ pele, utilizaram-se pontos isolados simples, com fio mononáilon $n^{0} 0$. Foi realizada sutura em bolsa de fumo ao redor do ânus de todos os animais, com fio poliglactina 910 nº 0, mantida por quatro dias. Aplicou-

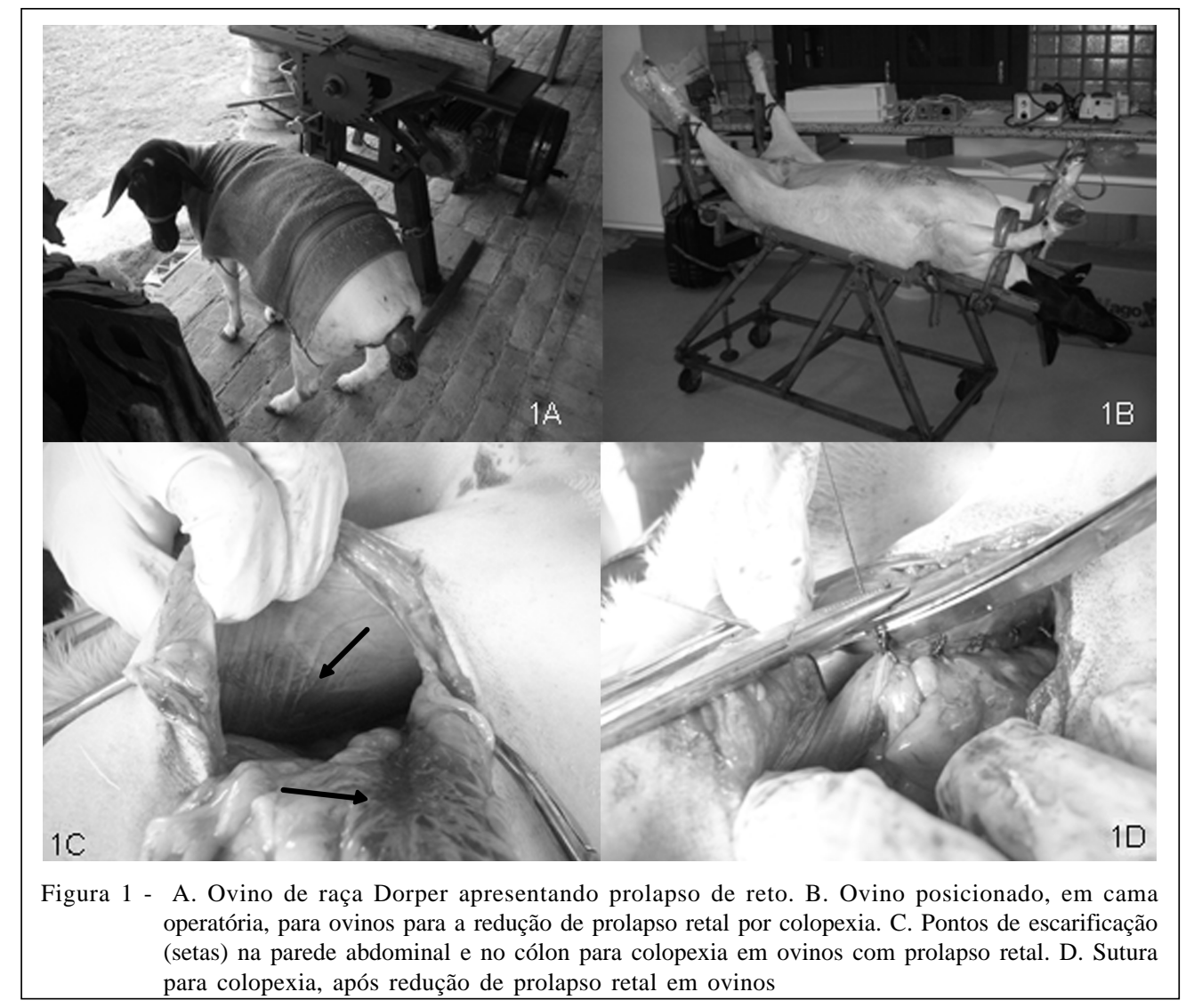

Ciência Rural, v.39, n.2, mar-abr, 2009. 
se flunixin meglumine ${ }^{\mathrm{d}}$, na dose de $1 \mathrm{mg} \mathrm{kg}^{-1}$, por via intramuscular, por três dias. O tratamento antibiótico prorrogou-se por mais sete dias com gentamicina na mesma dose que foi utilizada na profilaxia antibiótica.

Os animais foram avaliados, aos dois, quinze e trinta dias, sendo que sete deles foram acompanhados por período de tempo maior, variando até um ano. A avaliação foi basicamente clínica, observando-se comportamento, possíveis sinais clínicos de infecção, ocorrência de recidiva, apetite, a ferida cutânea e outras eventuais complicações do procedimento cirúrgico.

\section{RESULTADOS}

O tempo, desde o aparecimento do prolapso até a intervenção cirúrgica, variou de dois dias até um mês, sendo que melhores resultados foram obtidos, quando o prolapso foi reduzido em tempo menor. Dos trinta animais operados, três vieram a óbito, um macho e uma fêmea no quarto dia de pós-operatório e uma fêmea, no sétimo. Três animais que tiveram recidiva foram sacrificados, sendo um deles idoso, com recidiva aos 15 dias após a cirurgia e outros dois animais por se encontrarem bastante debilitados.

No mesmo dia da intervenção cirúrgica, um animal do grupo, que recebeu solução de Ringer com lactato na cavidade abdominal, teve ruptura dos pontos da parede abdominal, condição esta que demandou uma reintervenção cirúrgica para sua correção. No segundo dia de pós-operatório, todos os animais apresentavam-se em bom estado físico e sem recidiva do prolapso, alimentando-se bem, observando-se um edema na linha de sutura. Um dos ovinos apresentou hérnia incisional com cinco dias de pós-operatório. Aos 15 dias após a cirurgia, cinco animais apresentaram recidiva do prolapso retal, e a colopexia foi refeita em três, sendo os outros dois sacrificados, com bom resultado em apenas um deles. Aos 30 dias de pósoperatório, um animal apresentou sinais de prolapso retal e os outros dezenove (63,33\%) estavam em ótimo estado físico, sem sinais de prolapso. Após seis meses, sete animais foram reavaliados, e nenhum deles apresentava problemas relacionados à cirurgia ou recidivas.

\section{DISCUSSÃO}

Segundo SCHOENIAN (2006), o tratamento do prolapso de reto em ovinos é custo-proibitivo, porém os animais da raça Dorper relatados neste artigo possuíam alto valor zootécnico e representavam investimento muito grande de seus proprietários, o que justificou a intervenção corretiva. Os animais da raça
Dorper possuem corte de cauda curta, fator relacionado por THOMAS et al. (2003) e BULGIN (2006) como predisponente para a prevalência do prolapso de reto em animais dessa espécie, pois ovinos da raça Santa Inês, criados no mesmo tipo de manejo, não apresentam essa afecção, e são ovinos que não possuem corte de cauda.

Neste presente estudo, o número de fêmeas acometidas (87\%) foi muito superior ao número de machos (13\%), corroborando os achados de THOMAS et al. (2003), o que, segundo BULGIN (2006), se deve, provavelmente, à maior quantidade de gordura encontrada na região pélvica das fêmeas.

Alguns animais deste relato foram submetidos à eutanásia ou tiveram recidiva do prolapso, o que parece estar relacionado ao fato de seus proprietários demorarem muito tempo para solicitarem a assistência veterinária, a ponto de esses animais já estarem debilitados e seu tecido intestinal se apresentar friável e edemaciado. Isso se confirmou com o maior índice de sucesso, quando os animais apresentavam prolapso há menos de duas semanas, podendo-se observar ainda que, após a primeira recidiva, o índice de sucesso diminuiu drasticamente.

A anestesia utilizada neste estudo mostrouse satisfatória para a realização da colopexia. Embora SCOTT \& GESSERT (1997) tenham utilizado e indiquem os dois fármacos por via epidural, a utilização da xilazina por via intramuscular, complementada pela lidocaína epidural, mostrou-se alternativa adequada.

O tipo de amputação indicado por SCHOENIAN (2006) não pareceu aos autores deste estudo muito humanitário, uma vez que uma faixa elástica é colocada comprimindo os vasos intestinais, e, dessa forma, os animais passam por processo de necrose, perdendo a parte prolapsada do intestino, o que denota um grau de dor eticamente inaceitável. A utilização da colopexia, por sua vez, é tratamento bastante aceito do ponto de vista ético, desde que o animal esteja devidamente anestesiado.

Treze animais receberam a infusão intraabdominal de $500 \mathrm{~mL}$ de solução de Ringer com lactato, pois pertenciam a um centro de reprodução e os veterinários responsáveis pelo centro acreditavam que, com esse procedimento, ocorreriam menos aderências principalmente na região uterina, por isso, eles solicitaram. Nesses ovinos, no entanto, percebeu-se que a tensão no abdômen aumentava muito e, após um deles apresentar deiscência da ferida, poucas horas após a sutura, optou-se por evitar o procedimento, não ocorrendo outras deiscências da ferida cirúrgica.

Outro aspecto observado é que os animais que receberam infusão de solução na cavidade 
abdominal tiveram índice maior de recidiva, provavelmente, devido à diluição da fibrina na área de pexia, o que dificultou a aderência do intestino à cavidade abdominal. Segundo ZIMMERMANN et al. (2006), a lavagem da cavidade abdominal remove resíduos necróticos, exudatos e fibrinas. Para lavagem da cavidade abdominal, foi escolhida a solução de Ringer com lactato, pelo fato de ser solução menos agressiva, por ser balanceada em eletrólitos e possuir pH próximo da neutralidade (SCHNEIDER et al., 1988).

A colopexia, com prévia escarificação da parede abdominal e do tecido intestinal, foi realizada, conforme recomenda FOSSUM (2005), pois assegura maior grau de aderência. O fio de escolha foi o poliglactina 910, um fio absorvível, que se mostrou muito efetivo em relação à confecção da sutura, à semelhança das afirmações de BRUN et al. (2004), que a utilizaram em cães.

A sutura em bolsa de fumo adaptada, após a redução por colopexia, foi usada apenas como adjuvante temporário e não como um tipo de tratamento como sugere BULGIN (2006).

\section{CONCLUSÃO}

A realização de colopexia é alternativa viável para o tratamento de prolapso retal em ovelhas da raça Dorper, porém recidivas e complicações são comuns.

\section{FONTES DE AQUSIÇÃO}

a - Rompum ${ }^{\circledR}$, Bayer, São Paulo, SP

b - Lidocaína Abbott 2\% com epinefrina ${ }^{\circledR}$. Abbott. São Paulo, SP

c - Gentamicina, Geyer Medicamentos S.A. Porto Alegre, RS

d - Banamine, Schering-Plough, Cotia, SP.

\section{REFERÊNCIAS}

BARROS, N.N. et al. Eficiência bioeconômica de cordeiros F1 Dorper x Santa Inês para produção de carne. Pesquisa Agropecuária Brasileira, v.40, n.8, p.825-831, 2005.

BRUN, M.V. et al. Avaliação de dois diferentes fios de sutura para colopexia incisional laparoscopia em cães. Estudo experimental. Brazilian Journal of Veterinary Research and Animal Science, v.41, p.154-161, 2004.

BULGIN, M.S. Tail docks. United Suffolk Sheep Association. Caldwell, Idaho, 05 de janeiro de 2006. Capturado em 16 de maio de 2007. Online. Disponível na internet: http:/ /u-s-s-a.org/Dec05Jan06Health.htm.

CLOETE, S.W.P. et al. Productive performance of Dorper sheep. Small Ruminant Research, v.36, p.119-135, 2000.
COLODO, J.C.N. et al. Influência de fatores ambientais nas características de desempenho ponderal em ovinos da raça Santa Inês. In: SIMPÓSIO DA SOCIEDADE BRASILEIRA DE MELHORAMENTO ANIMAL, 2004, Pirassununga, SP. Capturado em 16 de maio de 2007. Online. Disponível na internet: http://www.sbmaonline.org.br/anais/v/trabalhos/ ovinos/ov002.pdf.

CORMAN, ML. Rectal prolapse. In: CORMAN M.L, Colon \& rectal surgery. 3.ed. Philadelphia: JB Lippincott, 1993. p.293-336.

FOSSUM, T.W. Cirurgia de pequenos animais. 2.ed. São Paulo: Roca, 2005. 1390p.

GUIMARÃES FILHO, C. et al. Sistemas de produção de carnes caprina e ovina no semi-árido nordestino. In: SIMPÓSIO INTERNACIONAL SOBRE CAPRINOS E OVINOS DE CORTE, 2000, João Pessoa, PB. Anais... João Pessoa: EMEPAPB, 2000. p.21-34.

INSTITUTO BRASILEIRO DE GEOGRAFIA E ESTATÍSTICA. Censo Agropecuário 1995-1996. Rio de Janeiro, 1996. Capturado em 16 de maio de 2007. On line. Disponível na internet: http://www.sidra.ibge.gov.br/bda/tabela/ protabl.asp? $\mathrm{z}=\mathrm{t} \& \mathrm{o}=21 \& \mathrm{i}=\mathrm{P}$.

MATTHIESEN, D.T.; MARRETA, S.M. Afecções do ânus e reto. In: SLATTER, D. Manual de cirurgia de pequenos animais. 2.ed. São Paulo: Manole, 1998. V.2, cap.46, p.760780 .

NOTTER, D.R. et al. Growth and carcass characteristics of lambs sired by Dorper and Dorset rams. Journal of Animal Science, v.82, p.1323-1328, 2004.

SCHNEIDER, R.K. et al. Response of pony peritoneum to four peritoneal lavage solutions. American Journal of Veterinary Research. v.49, p.889-894, 1988.

SCHOENIAN, S. Rectal prolapse: a complex problem with many contributing factors. Maryland small ruminant page. Maryland, 04 de novembro de 2006. Capturado em 16 de maio de 2007. Online. Disponível na Internet: http:// www.sheepandgoat.com/articles/rectalprolapse.html.

SCOTT, P.R.; GESSERT M.E. Management of post-partum cervical uterine or rectal prolapses in ewes using caudal epidural xylazine and lignocaine injection. Veterinary Journal, v.153, p.115-116, 1997.

SOUSA, W.H.; LEITE, P.R.M. Ovinos de corte: a raça Dorper. João Pessoa: Emepa-PB, 2000. 75p.

THOMAS, D.L. et al. Length of docked tail and the incidence of rectal prolapse in lambs. Journal of Animal Science, v.81, p.2725-2732, 2003.

ZIMMERMANN, M. et al. Peritonite em cães. Ciência Rural, v.36, n.5, p.1655-1663, 2006. 